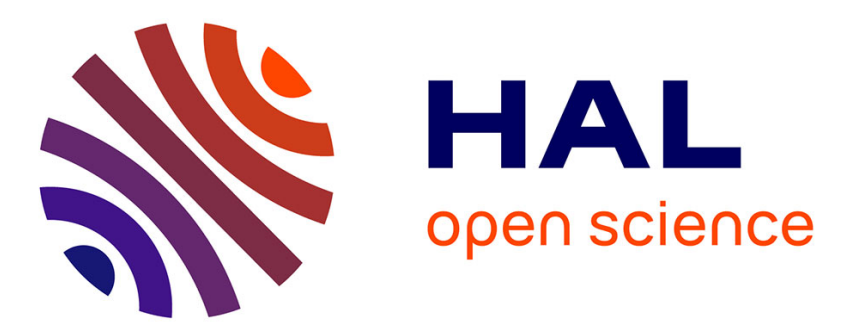

\title{
Interval Modeling and Robust Control of Piezoelectric Microactuators.
}

\author{
Sofiane Khadraoui, Micky Rakotondrabe, Philippe Lutz
}

\section{To cite this version:}

Sofiane Khadraoui, Micky Rakotondrabe, Philippe Lutz. Interval Modeling and Robust Control of Piezoelectric Microactuators.. IEEE Transactions on Control Systems Technology, 2012, 20 (2), pp.486-494. 10.1109/TCST.2011.2116789 . hal-00799345

\section{HAL Id: hal-00799345 \\ https://hal.science/hal-00799345}

Submitted on 18 Mar 2013

HAL is a multi-disciplinary open access archive for the deposit and dissemination of scientific research documents, whether they are published or not. The documents may come from teaching and research institutions in France or abroad, or from public or private research centers.
L'archive ouverte pluridisciplinaire HAL, est destinée au dépôt et à la diffusion de documents scientifiques de niveau recherche, publiés ou non, émanant des établissements d'enseignement et de recherche français ou étrangers, des laboratoires publics ou privés. 


\title{
Interval Modeling and Robust Control of Piezoelectric Microactuators
}

\author{
Sofiane Khadraoui, Micky Rakotondrabe, Member, IEEE and Philippe Lutz, Member, IEEE
}

\begin{abstract}
Microsystems are very sensitive to environmental disturbances (thermal variation, surrounding vibration, microobjects in contact with them, etc.) and they are often subjected to small degradation or their behaviors are often affected during the functioning. As a result, their parameters often change during the micromanipulation, microassembly or measurement tasks and the accuracy or even the stability may be lost. For that, robust control laws should be introduced to control them and to ensure the performance.

$H_{\infty}$ and $\mu$-synthesis approaches were the classical robust techniques used to control microsystems. They are undeniably efficient but they lead to high-order controllers that are sometimes inconvenient for real-time embedded systems. In this paper, by the means of interval numbers that are used to characterize the uncertain parameters, we propose a method to synthesize simple controllers ensuring robust performance for microsystems. The controller synthesis is formulated as a set-inclusion problem. The main advantages of the proposed method are the ease of modeling the uncertain parameters thanks to intervals and the simplicity and low-order of the derived controllers. The method is afterwards applied to model and control piezoelectric microactuators and the experimental results show its efficiency. Finally, using the $H_{\infty}$ technique, we also demonstrate numerically the performance robustness of the closed-loop with the designed controller.
\end{abstract}

Index Terms-Piezoelectric microactuators, Controller design, Robust performance, Parametric uncertainty, Interval systems.

\section{INTRODUCTION}

Due to their small sizes, microsystems are very sensitive to environmental disturbances (temperature, vibrations, etc.) and to the interaction and contact with surrounding systems (manipulated objects, other microsystems). Microgrippers - usually employed for micro/nanopositioning, micro/nanomanipulation and microassembly - are not exempt to such characteristics. During the manipulation of a microobject for instance, the actuators behaviors are affected and the parameters of their models are modified. It is therefore necessary to use robust controllers to ensure their performance during this functioning.

A microgripper is based on two cantilevers, called microactuators, taht are made of smart materials [1] [2]. Piezoelectric materials are the most used because of the high resolution, high bandwidth and high force density that they can offer [3]. While one of the two piezoelectric cantilevers (piezocantilevers) is controlled on position, the second one

Initial submission: september 2010 .

The authors are with the Automatic Control and Micro-Mechatronic Systems Department, AS2M.

FEMTO-ST Institute, UMR CNRS 6174/UFC/ENSMM/UTBM

24 rue Alain Savary, Besançon 25000, France.

e-mail: \{ sofiane.khadraoui,mrakoton,plutz\}@ femto-st.fr

phone: 0033381402 892, fax: 0033381402809

Paper type: brief paper. is controlled on manipulation force. To reach the required performance, the design of control laws for both actuators needs adapted models. In order to have simpler models but which account the characteristics of the piezocantilevers, linear models with uncertainties were used. Classical robust control laws such as $H_{2}, H_{\infty}$ and $\mu$-synthesis have been therefore applied to control each microactuator [4] [5]. The efficiency of these control methods is proved in several applications (SISO and MIMO systems) while its major disadvantage is the derivation of high-order controllers which are time consuming and which limit their embedding possibilities, particularly for embedded microsystems. A possible alternative to classical robust control laws is the use of interval analysis where the uncertain parameters of the transfer function or of the state-space models to be controlled are bounded by intervals. The principle is therefore based on the combination of the interval arithmetic and a linear control theory. In addition to its principle simplicity to model the uncertain parameters, the main advantage is the derivation of low order controllers.

The first idea on interval arithmetic has been proposed in 1924 by Burkill and in 1931 by Young, then later in 1966 with R.E. Moore's works [6]. Since, several applications on interval analysis have been raising. In [7] [8], the authors have proposed guaranteed parameters estimation based on the SIVIA algorithm (Set Inversion Via Interval Analysis). In [9] [10] [11], the stability analysis of a closed-loop scheme with a given controller was proposed using the Routh's criteria and/or the Kharitonov's theorem. Concerning the design of controller, [12] proposed an approach of state feedback control combined with intervals for the model parameters to synthesize a controller that ensures the stability. In [13], a PID controller that ensures robust performance was proposed by using a set-inclusion problem. The method was limited to interval second order system. Chen and Wang [14] also proposed a robust method to control interval systems. In their work, two controllers were necessary: a robust controller stabilizing the feedback first, and then a pre-filter to ensure the wanted performance. Li et al. [15] proposed a control algorithm prediction-based interval model that was efficiently applied to a welding process. In our previous work [17], a robust controller for interval systems with zero-order numerator was proposed. Its main advantage relative to the other existing works is that the order of the system's denominator is not limited and the derived controller has a low-order. This previous work also proved that interval analysis and related controller design can be very promising for modeling and control microsystems where the models parameters are subject to change during functioning. 
In this paper, we propose to design robust control law for piezoelectric microactuators modeled by parametric uncertain systems, where interval modeling [16] is introduced to characterize the uncertain parameters. Contrary to the previous work [17], the approach in this paper is extended to general transfer function, i.e. no limitation on the orders of both numerator and denominator. Despite its limitation to SISO parametric uncertain systems, the proposed approach derives a low order controller that is necessary for real-time embedded systems. In addition to the experimental results which prove the efficiency of the designed controller, a performance analysis is also performed using the classical $H_{\infty}$ tool.

The paper is organized as follows. In section-II, we present the preliminaries related to interval analysis and systems. Section-III is dedicated to the controller design. The experimental results on piezoelectric microactuators are detailed in section-IV. Finally, in section-V we present the performance analysis based on the $H_{\infty}$ approach.

\section{MATHEMATICAL PRELIMINARIES}

\section{A. Basic Terms and Concepts on intervals}

More details on the preliminaries given here can be found in [6] or [9]. A closed interval $[x]$ is such that $[x]=\left[x^{-}, x^{+}\right]=$ $\left\{x \in R / x^{-} \leq x \leq x^{+}\right\}$, where $x^{-}$and $x^{+}$are the endpoints. We say that $[x]$ is degenerate if $x^{-}=x^{+}$. By convention, a degenerate interval $[a, a]$ can be denoted by $a$. The width of an interval $[x]$ is $w([x])=x^{+}-x^{-}$, the midpoint is $\operatorname{mid}([x])=$ $\frac{x^{+}+x^{-}}{2}$ and the radius is $\operatorname{rad}([x])=\frac{x^{+}-x^{-}}{2}$.

The operation between two intervals gives an interval containing all the operation results between all pairs of numbers in the two intervals. If we have two intervals $[x]=\left[x^{-}, x^{+}\right]$ and $[y]=\left[y^{-}, y^{+}\right]$and a law $\circ \in\{+,-, ., /\}$, we can write $[x] \circ[y]=\{x \circ y \mid x \in[x], y \in[y]\}$. The quotient is particularly defined by $[x] /[y]=[x] .\left[1 / y^{+}, 1 / y^{-}\right], 0 \notin[y]$.

The intersection of two intervals $[x] \cap[y]$ is the interval defined by $[x] \cap[y]=\emptyset$ if $y^{+}<x^{-}$or $x^{+}<y^{-}$. Otherwise, the result is $[x] \cap[y]=\left[\max \left\{x^{-}, y^{-}\right\}, \min \left\{x^{+}, y^{+}\right\}\right]$. In the latter case, the union of $[x]$ and $[y]$ is also an interval $[x] \cup[y]=\left[\min \left\{x^{-}, y^{-}\right\}, \max \left\{x^{+}, y^{+}\right\}\right]$.

When $[x] \cap[y]=\emptyset$, the union of the two intervals is not an interval. For that, the interval hull is defined: $[x] \underline{\cup}[y]=$ $\left[\min \left\{x^{-}, y^{-}\right\}, \max \left\{x^{+}, y^{+}\right\}\right]$.

It is verified that: $[x] \cup[y] \subseteq[x] \underline{\cup}[y]$ for any two intervals $[x]$ and $[y]$.

\section{B. Interval system}

Definition 1: Parametric uncertain systems can be modeled by interval systems. An interval system denoted $[G](s,[a],[b])$ is a family of systems:

$$
[G](s)=\frac{\sum_{j=0}^{m}\left[b_{j}\right] s^{j}}{\sum_{i=0}^{n}\left[a_{i}\right] s^{i}}=\left\{\begin{array}{l}
\frac{\sum_{j=0}^{m} b_{j} s^{j}}{\sum_{i=0}^{n} a_{i} s^{i}} \mid b_{j} \in\left[b_{j}^{-}, b_{j}^{+}\right] \\
a_{j} \in\left[a_{j}^{-}, a_{j}^{+}\right]
\end{array}\right\}
$$

such as: $[b]=\left[\left[b_{0}\right], \ldots,\left[b_{m}\right]\right]$ and $[a]=\left[\left[a_{0}\right], \ldots,\left[a_{n}\right]\right]$ are two boxes of interval parameters and $s$ the Laplace variable.
The following lemma which is a result for interval functions inclusion is due to [6].

Lemma 1: (Containment Theorem) Given $[F]([x])$ a rational expression in the interval variables $[x]=\left[\left[x_{1}\right], \ldots,\left[x_{n}\right]\right]$. Let $[y]=\left[\left[y_{1}\right], \ldots,\left[y_{n}\right]\right]$ be a box of interval variables. If $[y] \subseteq$ $[x]$, i.e. $\left[y_{1}\right] \subseteq\left[x_{1}\right], \ldots,\left[y_{n}\right] \subseteq\left[x_{n}\right]$, then $[F]([y]) \subseteq[F]([x])$.

Proof: see [6]

The following theorem is a straightforward consequence of Lemma 1.

Theorem 1: Given two stable interval transfers $\left[G_{1}\right](s,[\alpha],[\beta])$ and $\left[G_{2}\right](s,[\gamma],[\lambda])$ defined as in Definition 1 and having the same structure (same degree for their numerators, idem for their denominators). If $[\alpha] \subseteq[\gamma]$ and $[\beta] \subseteq[\lambda]$, then $\left[G_{1}\right](s,[\alpha],[\beta]) \subseteq\left[G_{2}\right](s,[\gamma],[\lambda])$.

Proof: Noting that $s=[s, s]=[s]$ and $[s] \subseteq[s]$, and applying Lemma 1 with $[F]([x])=\left[G_{2}\right](s,[\gamma],[\lambda])$ and $[F]([y])=\left[G_{1}\right](s,[\alpha],[\beta])$, where $[x]=[[s],[\gamma],[\lambda]]$ and $[y]=[[s],[\alpha],[\beta]]$, we have:

$$
[y] \subseteq[x] \Rightarrow[F]([y]) \subseteq[F]([x])
$$

which leads for any $s$ to:

$$
\left\{\begin{array}{l}
{[\alpha] \subseteq[\gamma]} \\
{[\beta] \subseteq[\lambda]}
\end{array} \Rightarrow\left[G_{1}\right](s,[\alpha],[\beta]) \subseteq\left[G_{2}\right](s,[\gamma],[\lambda])\right.
$$

\section{Performance of interval systems}

The following theorem is considered to define the time and frequency domains performance inclusion for interval systems.

Theorem 2: Given two stable interval transfers $\left[G_{1}\right](s,[\alpha],[\beta])$ and $\left[G_{2}\right](s,[\gamma],[\lambda])$ with the same structure. If $[\alpha] \subseteq[\gamma]$ and $[\beta] \subseteq[\lambda]$, then the time and the frequency domains responses of $\left[G_{1}\right](s,[\alpha],[\beta])$ are bounded by those of $\left[G_{2}\right](s,[\gamma],[\lambda])$. These responses define the time and frequency performance respectively.

Proof: 1) Temporal performance inclusion proof. Consider the two interval transfers $\left[G_{1}\right](s,[\alpha],[\beta])$ and $\left[G_{2}\right](s,[\gamma],[\lambda])$ which have the same structure. If $[\alpha] \subseteq[\gamma]$ and $[\beta] \subseteq[\lambda]$, then by applying Theorem 1 we have:

$$
\left[G_{1}\right](s,[\alpha],[\beta]) \subseteq\left[G_{2}\right](s,[\gamma],[\lambda])
$$

As $e^{s t}$ is a degenerate interval for any $s$ (Laplace variable) and any $t$ (time variable), the inclusion (2) is equivalent to:

$$
\left[G_{1}\right](s,[\alpha],[\beta]) e^{s t} \subseteq\left[G_{2}\right](s,[\gamma],[\lambda]) e^{s t}
$$

According to the interval properties (see [6]), applying the integration to (3) will not modify the inclusion:

$$
\int_{-\infty}^{+\infty}\left[G_{1}\right](s,[\alpha],[\beta]) e^{s t} d s \subseteq \int_{-\infty}^{+\infty}\left[G_{2}\right](s,[\gamma],[\lambda]) e^{s t} d s
$$

(4) can be rewritten as follows:

$$
\frac{1}{2 \pi j} \int_{-\infty}^{+\infty}\left[G_{1}\right](s,[\alpha],[\beta]) e^{s t} d s \subseteq \frac{1}{2 \pi j} \int_{-\infty}^{+\infty}\left[G_{2}\right](s,[\gamma],[\lambda]) e^{s t} d s
$$

The two terms in (5) represent the inverse Laplace trans ${ }^{2}$ forms of $\left[G_{1}\right](s,[\alpha],[\beta])$ and $\left[G_{2}\right](s,[\gamma],[\lambda])$ that describe the time responses $\left[g_{1}\right](t)$ and $\left[g_{2}\right](t)$ respectively, so: 


$$
\left[g_{1}\right](t) \subseteq\left[g_{2}\right](t)
$$

2) Frequential performance inclusion proof. This demonstration is obvious when using the Theorem 1 . Indeed, the inclusion (2) holds for any $s$, in the case where $s=j w$, we obtain:

$$
\left[G_{1}\right](j w,[\alpha],[\beta]) \subseteq\left[G_{2}\right](j w,[\gamma],[\lambda])
$$

which states the inclusion of the frequency responses of the two terms.

\section{COMPutation OF THE CONTROLleR}

In this section, we design a robust controller by using the interval analysis.

\section{A. Problem statement}

Given an uncertain system $[G](s,[a],[b])$ to be controlled by a controller $[C](s)$ (Fig. 1). This controller must ensure some given performance for the closed-loop whatever the parameters $a$ and $b$ ranging in $[a]$ and $[b]$ respectively are. Let $\left[H_{c l}\right](s,[p],[q])$ denote the closed-loop transfer. On the other hand, in the sequel, let the system $[G](s,[a],[b])$ be denoted by:

$$
[G](s,[a],[b])=\frac{1+\sum_{j=1}^{m}\left[b_{j}\right] s^{j}}{\sum_{i=0}^{n}\left[a_{i}\right] s^{i}}
$$

Such as $[a]=\left[\left[a_{0}\right], \ldots,\left[a_{n}\right]\right]$ and $[b]=\left[\left[b_{0}\right], \ldots,\left[b_{m}\right]\right], m \leq n$ and $\left[b_{0}\right]=1$.

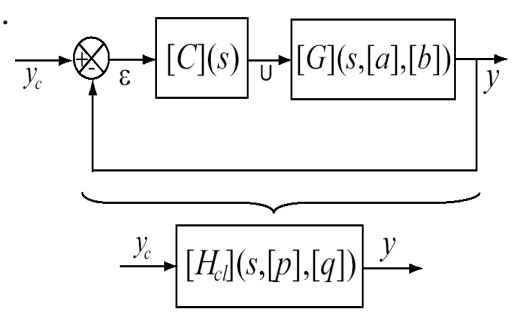

Fig. 1. Closed-loop transfer $H_{c l}$.

\section{B. Computation of the closed-loop model}

Let us define the controller $[C](s,[\theta])$ with a prior knowledge on its structure as follows:

$$
[C](s,[\theta])=\frac{\sum_{j=0}^{l}\left[d_{j}\right] s^{j}}{\sum_{i=0}^{k}\left[c_{i}\right] s^{i}}
$$

where $\theta=[[c],[d]]$ represents the interval parameters vector of the controller. We have $l \leq k$.

The closed-loop model can be computed using the interval model (8) and the controller (9) as follows:

$$
\left[H_{c l}\right](s,[a],[b],[\theta])=\frac{1}{\frac{1}{[C](s,[\theta])[G](s,[a],[b])}+1}
$$

After replacing $[G](s,[a],[b])$ and $[C](s,[\theta])$, we get:

$$
\left[H_{c l}\right](s)=\frac{\left(1+\sum_{j=1}^{m}\left[b_{j}\right] s^{j}\right) \cdot \sum_{j=0}^{l}\left[d_{j}\right] s^{j}}{\sum_{i=0}^{n}\left[a_{i}\right] s^{i} \cdot \sum_{j=0}^{k}\left[c_{i}\right] s^{i}+\left(1+\sum_{j=1}^{m}\left[b_{j}\right] s^{j}\right) \cdot \sum_{j=0}^{l}\left[d_{j}\right] s^{j}}
$$

After developing (11) and factorizing the last coefficient of the numerator, we obtain:

$$
\left[H_{c l}\right](s,[p],[q])=\frac{1+\sum_{j=1}^{e}\left[q_{j}\right] s^{j}}{\sum_{i=0}^{r}\left[p_{i}\right] s^{i}}
$$

Where $e=m+l$ and $r=n+k$ and where the boxes $[q]$ and $[p]$ are function of the boxes $[a],[b],[c]$ and $[d]$.

\section{Controller derivation}

The aim consists to compute the set parameters of the controller (9) that will ensure given specifications described by an interval reference model. The reference model, denoted by $[H]$, defines a family of wanted closed-loop behaviors. In other words, the main objective consists to find the set $\Theta$ of controller parameter vector for which robust performance holds:

$$
\Theta:=\left\{\theta \in[\theta] \mid\left[H_{c l}\right](s,[p],[q]) \subseteq[H](s)\right\}
$$

In order to check the inclusion $\left[H_{c l}\right](s,[p],[q]) \subseteq[H](s)$ by applying the parameter by parameter inclusion as given in Theorem 1, the interval reference model $[H]$ must have the same structure than $\left[H_{c l}\right]$ described in (12). Let us consider the following interval reference model:

$$
[H](s,[w],[x])=\frac{1+\sum_{j=1}^{e}\left[x_{j}\right] s^{j}}{\sum_{i=0}^{r}\left[w_{i}\right] s^{i}}
$$

Remark 1: The boxes of interval parameters $[w]$ and $[x]$ are known because they are computed from the specifications.

According to Theorem 2, if we have:

$$
\begin{array}{ll}
{\left[q_{j}\right] \subseteq\left[x_{j}\right],} & \text { for } \quad j=1, \ldots, e \\
{\left[p_{i}\right] \subseteq\left[w_{i}\right],} & \text { for } \quad i=0, \ldots, r
\end{array}
$$

then we obtain $\left[H_{c l}\right] \subseteq[H]$ and therefore the performance of $\left[H_{c l}\right]$ is included in those of $[H]$. As a result, the computed controller $[C]$ will effectively ensure the performance for any system $G$ in the interval model $[G]$.

The computation problem (13) of the set parameters $\Theta$ is reduced to the following problem:

$$
\Theta:=\left\{\begin{array}{l|l}
\theta \in[\theta] \mid \begin{array}{c}
{\left[q_{j}\right]([\theta]) \subseteq\left[x_{j}\right], \forall j=1, \ldots, m+1} \\
{\left[p_{i}\right]([\theta]) \subseteq\left[w_{i}\right], \forall i=0, \ldots, n+1}
\end{array}
\end{array}\right\}
$$

The problem of finding the set parameters of the controller ensuring (16) is known as a set-inclusion problem which can be solved using set inversion algorithms. The set inversion operation consists to compute the reciprocal image of a compact set called subpaving. The set-inversion algorithm SIVIA (more details are given in [7], [9]) allows to approximate with subpavings the set solution $\Theta$ described in (16). This approximation is realized with an inner and outer subpavings, 
respectively $\underline{\Theta}$ and $\bar{\Theta}$, such that $\underline{\Theta} \subset \Theta \subset \bar{\Theta}$. The subpaving $\Theta$ corresponds to the controller parameter vector for which the problem (16) holds. If $\Theta=\emptyset$, then it is guaranteed that no solution exists for (16).

We give in Table I the recursive SIVIA algorithm allowing to solve the control problem (16) with guaranteed solutions. SIVIA algorithm requires a search box $[\theta](0)$ (possibly very large) also called initial box within which $\bar{\Theta}$ is guaranteed to belong. The inner and outer subpavings $(\underline{\Theta}$ and $\bar{\Theta})$ are initially empty. $\epsilon$ represents the wanted accuracy of computation.

TABLE I

ALGORITHM SIVIA FOR SOLVING A SET-INVERSION PROBLEM [7], [9].

\begin{tabular}{|c|l|}
\hline & SIVIA(in: $[p],[q],[w],[x],[\theta], \epsilon$; inout: $\underline{\Theta}, \bar{\Theta})$ \\
\hline 1 & if $[[p]([\theta]),[q]([\theta])] \cap[[w],[x]]=\emptyset$ return; \\
2 & if $[[p]([\theta]),[q]([\theta])] \subset[[w],[x]]$ then \\
& $\{\underline{\Theta}:=\underline{\Theta} \bigcup[\theta] ; \bar{\Theta}:=\bar{\Theta} \bigcup[\theta]\}$ return; \\
4 & if width $([\theta])<\epsilon$ then $\{\bar{\Theta}:=\bar{\Theta} \bigcup[\theta]\} ;$ return; \\
5 & bisect $[\theta]$ into $L([\theta])$ and $R([\theta]) ;$ \\
6 & SIVIA $([p],[q],[w],[x], L([\theta]), \epsilon ; \underline{\Theta}, \bar{\Theta}) ;$ \\
& SIVIA $([p],[q],[w],[x], R([\theta]), \epsilon ; \underline{\Theta}, \bar{\Theta})$. \\
\hline
\end{tabular}

In most cases, it is more interesting to compute an inner subpaving $\underline{\Theta}$ for which one is sure that $\underline{\Theta}$ is included in the set solution $\Theta$, i.e. $\Theta \subset \Theta$. However, when no inner subpaving exists i.e. $\underline{\Theta}=\emptyset$, it is possible to choose parameters inside the outer subpaving, i.e. choose $\theta \in \bar{\Theta}$.

Remark 2: The number of unknown parameters (see (9)) are $l+k+2$ while the number of inclusions is $r+e+1$. Since $e=m+l$ and $r=n+k$, we can write $r+e+1 \geq l+k+2$. Therefore, there are more inclusions than unknown variables. So, the set solution $\Theta$ is given by the intersection of the set solution of each inclusion in (16) as follows:

$$
\Theta=\bigcap_{i=1}^{r+e+1}(\text { set_sol })_{i}
$$

such as: $(\text { set_sol })_{i}$ is the set solution of the $i^{t h}$ inclusion.

Remark 3: The controller is said to be low-order since its order is independent from the complexity of the specifications, which is the case for $H_{\infty}$ based approach.

\section{CONTROL OF PIEZOCANTILEVERS}

The aim of this section is to apply the proposed method to control piezoelectric microactuators used in microgrippers. The actuators have a cantilever structure and are also called piezocantilevers. A piezocantilever is a cantilever based on one or more piezoelectric layers. In this paper, we especially use a unimorph piezocantilever with a rectangular cross-section. A unimorph piezocantilever is made up of one piezoelectric layer and one passive layer. We use a ceramic Lead-ZirconateTitanate (PZT) as piezoelectric material while Copper as passive one. When a voltage $U$ is applied to the piezolayer it contracts or expands and a deflection $\delta$ of the whole cantilever is obtained (Fig. 2).

Modeling and identification of microsystems are very delicate because of their small sizes, their fragility and the lack of convenient (accurate and high bandwidth) sensors to report precise measurements. These systems are also very sensitive to environmental disturbances (temperature, vibrations, manipulated objects, etc.). As a result, their behavior

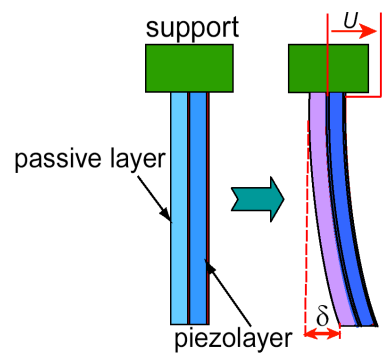

Fig. 2. Piezocantilever principle.

parameters may change during their functioning or during the tasks and the performance or even the stability may be lost. To account these characteristics, linear models with uncertainties and classical robust conrtol laws $\left(H_{\infty}\right)$ were applied and have proven their efficiency [18]. However, the derived controllers have high-order and are not often appropriate for embedding systems, which is often the case in several microsystems. In this paper, the uncertain parameters of piezocantilevers models are bounded by intervals. Afterwards, we apply the controller design previously presented to enhance their performance.

To derive $[G](s,[a],[b])$, we use two piezocantilevers with the same dimensions. Despite their similarity in dimensions, small differences of somes microns (due to the imprecision of the microfabrication process) generate non-negligible difference on their model parameters. So, instead of having a model with time-varying parameters during the experiment, we define from the two models of piezocantilevers one interval model. This interval model is further used to design a robust controller for both piezocantilevers.

\section{A. Presentation of the setup}

Fig. 3 shows the experimental setup which is composed of two unimorph piezocantilevers having nearly the same dimensions: $L \times b \times h=15 \mathrm{~mm} \times 2 \mathrm{~mm} \times 0.3 \mathrm{~mm}$, a Keyence optical sensor with $10 \mathrm{~nm}$ of resolution and used to measure the deflections of the two piezocantilevers, a computer-dSPACE hardware combined with the Matlab-Simulink software for the implementation of the controller (the refresh time is $0.2 \mathrm{~ms}$ ), and a high-voltage (HV) amplifier used to amplify the input voltage from the computer-dSPACE material.

\section{B. Modeling and identification}

The relation between the input voltage $U$ and the output deflection at the tip of a piezocantilever is [18]:

$$
\delta=G(s) U
$$

where, for us, the transfer functions $G_{1}(s)$ and $G_{2}(s)$ that model the two piezocantilevers must be identified.

A frequentiel identification is performed by applying a sine sweep signal to each piezocantilever [19]. We are interested to the first mode (resonance). For that, a second order model was chosen. After identification under matlab software, we obtain:

$$
\begin{aligned}
& G_{1}(s)=\frac{8.0313 \times 10^{-8} s^{2}+1.808 \times 10^{-4} s+1}{9.794 \times 10^{-8} s^{2}+5.24 \times 10^{-6} s+1.44} \\
& G_{2}(s)=\frac{7.042 \times 10^{-8} s^{2}+1.809 \times 10^{-4} s+1}{8.802 \times 10^{-8} s^{2}+5.364 \times 10^{-6} s+1.291}
\end{aligned}
$$




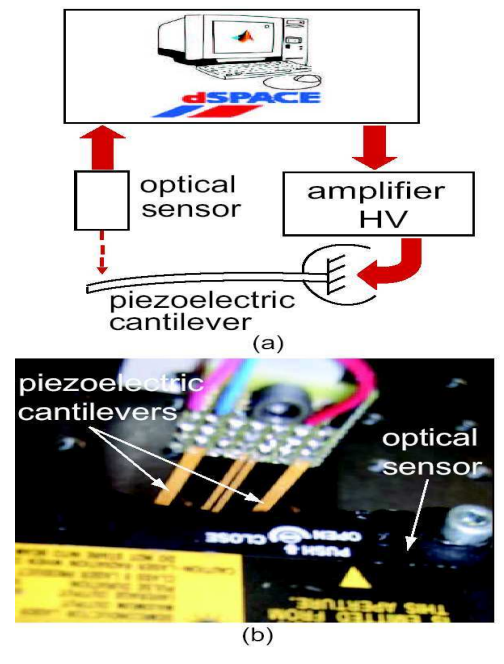

Fig. 3. The experimental setup.

\section{Derivation of the interval model}

Let us rewrite each model $G_{i}(s)(i=1,2)$ as follows:

$$
G_{i}(s)=\frac{b_{2 i} s^{2}+b_{1 i} s+b_{0 i}}{a_{2 i} s^{2}+a_{1 i} s+a_{0 i}}
$$

The interval model $[G](s,[a],[b])$ which represents a family of piezocantilever models is derived using the two point models $G_{i}(s)$. Considering each parameter of $G_{1}(s)$ and the corresponding parameter in $G_{2}(s)$ as an endpoint of the interval parameter in $[G](s,[a],[b])$, we have:

such as:

$$
[G](s,[a],[b])=\frac{\left[b_{2}\right] s^{2}+\left[b_{1}\right] s+\left[b_{0}\right]}{\left[a_{2}\right] s^{2}+\left[a_{1}\right] s+\left[a_{0}\right]}
$$

$$
\begin{aligned}
& {\left[b_{2}\right]=\left[\min \left(b_{21}, b_{22}\right), \max \left(b_{21}, b_{22}\right)\right]} \\
& {\left[b_{1}\right]=\left[\min \left(b_{11}, b_{12}\right), \max \left(b_{11}, b_{12}\right)\right]} \\
& {\left[b_{0}\right]=\left[\min \left(b_{01}, b_{02}\right), \max \left(b_{01}, b_{02}\right)\right]} \\
& {\left[a_{2}\right]=\left[\min \left(a_{21}, a_{22}\right), \max \left(a_{21}, a_{22}\right)\right]} \\
& {\left[a_{1}\right]=\left[\min \left(a_{11}, a_{12}\right), \max \left(a_{11}, a_{12}\right)\right]} \\
& {\left[a_{0}\right]=\left[\min \left(a_{01}, a_{02}\right), \max \left(a_{01}, a_{02}\right)\right]}
\end{aligned}
$$

After the numerical application, we obtain:

$$
\begin{aligned}
& {\left[b_{2}\right]=[7.042,8.0313] \times 10^{-8}} \\
& {\left[b_{1}\right]=[1.808,1.809] \times 10^{-4}} \\
& {\left[b_{0}\right]=1} \\
& {\left[a_{2}\right]=[8.802,9.794] \times 10^{-8}} \\
& {\left[a_{1}\right]=[5.24,5.364] \times 10^{-6}} \\
& {\left[a_{0}\right]=[1.291,1.44]}
\end{aligned}
$$

In order to increase the stability margin and to ensure that the interval model really contains the models of the two piezocantilevers, we propose to expand the interval width of each parameter of (20) by $10 \%$. Finally, the interval model that will be used is:

Such as:

$$
[G](s,[a],[b])=\frac{\left[b_{2}\right] s^{2}+\left[b_{1}\right] s+1}{\left[a_{2}\right] s^{2}+\left[a_{1}\right] s+\left[a_{0}\right]}
$$

$$
\begin{aligned}
& {\left[b_{2}\right]=[6.992,8.08] \times 10^{-8}} \\
& {\left[b_{1}\right]=[1.807,1.809] \times 10^{-4}} \\
& {\left[a_{2}\right]=[8.753,9.844] \times 10^{-8}} \\
& {\left[a_{1}\right]=[5.234,5.37] \times 10^{-6}} \\
& {\left[a_{0}\right]=[1.283,1.448]}
\end{aligned}
$$

\section{Definition of the specifications}

The following specifications are provided for the closedloop. These specifications often correspond to the requirement in precise positioning tasks of microassembly or micromanipulation applications that use piezoelectric microgrippers.

- no overshoot,

- settling time $1 \mathrm{~ms} \leq t r_{5 \%} \leq 30 \mathrm{~ms}$,

- static error $|\varepsilon| \leq 1 \%$.

\section{E. Definition of the controller structure}

Any structure of the controller $[C](s)$ is possible with the proposed approach as long as Remark 2 is satisfied. In this example, we consider a $P I$ (proportional Integral) structure because of its low-order (two parameters) and its wide use in industry:

$$
[C]\left(s,\left[K_{p}\right],\left[K_{i}\right]\right)=\frac{\left[K_{p}\right] s+\left[K_{i}\right]}{s}
$$

where $K_{p}$ and $K_{i}$ are the proportional and the integral gains respectively.

\section{F. Computation of the closed-loop and of the reference model}

The general model of the closed-loop is given by (10). In our case, using the model in (21) and the interval controller in (22), the closed-loop has the following form:

$$
\left[H_{c l}\right](s,[p],[q])=\frac{\left[q_{3}\right] s^{3}+\left[q_{2}\right] s^{2}+\left[q_{1}\right] s+1}{\left[p_{3}\right] s^{3}+\left[p_{2}\right] s^{2}+\left[p_{1}\right] s+\left[p_{0}\right]}
$$

such as:

$$
\begin{aligned}
& {\left[q_{3}\right]=\frac{\left[K_{p}\right]\left[b_{2}\right]}{\left[K_{i}\right]}} \\
& {\left[q_{2}\right]=\frac{\left[K_{p}\right]\left[b_{1}\right]}{\left[K_{i}\right]}+\left[b_{2}\right]} \\
& {\left[q_{1}\right]=\frac{\left[K_{p}\right]}{\left[K_{i}\right]}+\left[b_{1}\right]} \\
& {\left[p_{3}\right]=\frac{\left[a_{2}\right]+\left[K_{p}\right]\left[b_{2}\right]}{\left[K_{i}\right]}} \\
& {\left[p_{2}\right]=\frac{\left[a_{1}\right]+\left[K_{p}\right]\left[b_{1}\right]}{\left[K_{i}\right]}+\left[b_{2}\right]} \\
& {\left[p_{1}\right]=\frac{\left[a_{0}\right]+\left[K_{p}\right]}{\left[K_{i}\right]}+\left[b_{1}\right]} \\
& {\left[p_{0}\right]=1}
\end{aligned}
$$

Concerning the reference model, its computation is carried out accordingly to the required specifications. Since there is no evershoot in the specifications (see Section IV-D), a first order model can be used for the reference model:

$$
[H](s,[K],[\tau])=\frac{[K]}{[\tau] s+1}
$$

where the parameters $[K]$ and $[\tau]$ define the static error and settling time respectively such that:

- $[K]=1+\varepsilon=[0.99,1.01]$,

- $[\tau]=\frac{\left[\operatorname{tr}_{5} \%\right]}{3}=[0.33 \mathrm{~ms}, 10 \mathrm{~ms}]$.

However, it is necessary that the reference model has the same structure than the closed-loop in (23) in order to apply the parameter by parameter inclusion as required in (16). Thus we add some poles and zeros far from the imaginary axis to (24):

$$
[H](s,[K],[\tau])=\frac{[K]\left(\frac{[\tau]}{10} s+1\right)^{3}}{([\tau] s+1) \cdot\left(\frac{[\tau]}{10} s+1\right)^{2}}
$$

which can also be rewritten as follows: 


$$
[H](s,[w],[x])=\frac{\left[x_{3}\right] s^{3}+\left[x_{2}\right] s^{2}+\left[x_{1}\right] s+1}{\left[w_{3}\right] s^{3}+\left[w_{2}\right] s^{2}+\left[w_{1}\right] s+\left[w_{0}\right]}
$$

such as:

$$
\begin{aligned}
& {\left[x_{3}\right]=0.001[\tau]^{3}} \\
& {\left[x_{2}\right]=0.03[\tau]^{2}} \\
& {\left[x_{1}\right]=0.3[\tau]} \\
& {\left[w_{3}\right]=\frac{0.01[\tau]^{3}}{[K]}} \\
& {\left[w_{2}\right]=\frac{0.21[\tau]^{2}}{[K]}} \\
& {\left[w_{1}\right]=\frac{1.2[\tau]}{[K]}} \\
& {\left[w_{0}\right]=\frac{1}{[K]}}
\end{aligned}
$$

\section{G. Condition to have the robust performance}

The controller defined in (22) ensures the required specifications in Section IV-D for the interval model (21) if the parameters $\left[K_{p}\right]$ and $\left[K_{i}\right]$ meet the following inclusions:

$$
\begin{aligned}
& \frac{\left[K_{p}\right]\left[b_{2}\right]}{\left[K{ }_{i}\right]} \subseteq 0.001[\tau]^{3} \\
& \frac{\left[K_{p}\right]\left[b_{1}\right]}{\left[K b_{i}\right]}+\left[b_{2}\right] \subseteq 0.03[\tau]^{2} \\
& \frac{\left[K_{p}\right]}{\left[K_{i}\right]}+\left[b_{1}\right] \subseteq 0.3[\tau] \\
& \frac{\left[a_{2}\right]+\left[K_{p}\right]\left[b_{2}\right]}{\left[K_{i}\right]} \subseteq \frac{0.01[\tau]^{3}}{[K]} \\
& \frac{\left[a_{1}\right]+\left[K_{p}\right]\left[b_{1}\right]}{\left[K_{i}\right]}+\left[b_{2}\right] \subseteq \frac{0.21[\tau]^{2}}{[K]} \\
& \frac{\left[a_{0}\right]+\left[K_{p}\right]}{\left[K_{i}\right]}+\left[b_{1}\right] \subseteq \frac{1.2[\tau]}{[K]} \\
& 1 \subseteq \frac{1}{[K]}
\end{aligned}
$$

\section{H. Numerical derivation of the controller}

Let us now compute the controller parameters that ensure the performance. The problem of finding the controller parameters that satisfy the inclusions given by (27) is a setinclusion problem. If we denote $S_{c}$ the set parameters of the controller that satisfies these conditions, $S_{c}$ can be solved using the SIVIA algorithm [7] if an initial box is provided. After the application of the SIVIA algorithm implemented in the Matlab-Software, with an initial box $\left[K_{p 0}\right] \times\left[K_{i 0}\right]=$ $[0.1,0.6] \times[0.001,500]$, we obtain the subpaving given in Fig. 4 . The dark colored subpaving $\left(S_{c}\right)$ corresponds to the set parameters $\left[K_{p}\right]$ and $\left[K_{i}\right]$ of the controller (22) that ensures the performance for the interval model.

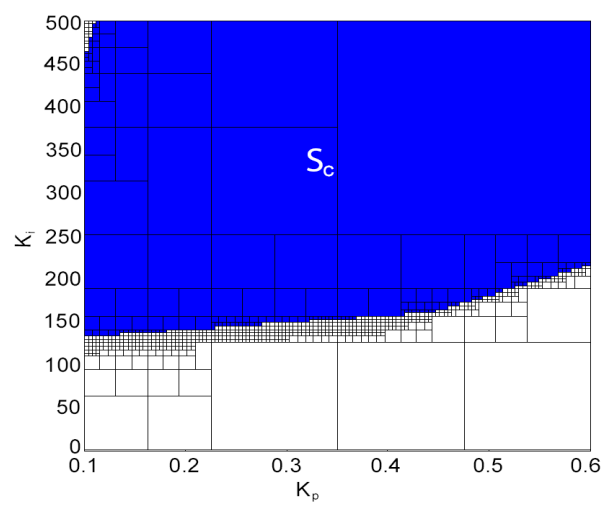

Fig. 4. Set solution of the parameters $\left[K_{p}\right]$ and $\left[K_{i}\right]$ ensuring the wanted performance.
Remark 4: We notice that any choice of the parameters $\left[K_{p}\right]$ and $\left[K_{i}\right]$ in the dark colored subpaving $S_{c}$ (see Fig. 4) satisfies the inclusion defined in (27) and consequently ensures specifications.

Remark 5: If the set-inclusion problem is not feasible, i.e. $S_{c}=\emptyset$, the initial box of the parameters must be changed and/or one must modify the controller structure and/or the specifications.

\section{Experimental results}

The controller $[C]\left(s,\left[K_{p}\right],\left[K_{i}\right]\right)$ is interval and is not directly implementable. Point parameters $K_{p}$ and $K_{i}$ within the set solution $S_{c}$ must be chosen and the corresponding point controller $C\left(s, K_{p}, K_{i}\right)=C(s)$ has to be implemented. In this example, we test two point controllers. We choose:

$$
\begin{aligned}
& C_{1}(s)=\frac{0.1 s+200}{s} \\
& C_{2}(s)=\frac{0.2 s+400}{s}
\end{aligned}
$$

These two controllers are applied to the two piezocantilevers. First, a step response analysis is performed. Fig. 5 shows the experimental results when a step reference of $20 \mu \mathrm{m}$ is applied. To check that the experimental results with the two controllers $C_{1}(s)$ and $C_{2}(s)$ satisfy the specifications, the temporal envelope of the interval reference model $[H](s,[K],[\tau])$ is also plotted in the same figure. The envelope of the desired behaviour is defined by the responses of two tranfer functions $H_{1}(s)$ and $H_{2}(s)$ such as: 1) $H_{1}(s)$ has the minimal time constant $\tau=0.33 \mathrm{~ms}$ and the maximal static gain $K=1.01$, 2) and $H_{2}(s)$ has the maximal time constant $\tau=10 \mathrm{~ms}$ and the minimal static gain $K=0.99$.

As shown in Fig. 5, the controllers have played their roles because the closed-loop piezocantilevers satisfy the specifications. Indeed, the experimental settling times are about $t r_{1}=17.7 \mathrm{~ms}$ and $t r_{2}=7 \mathrm{~ms}$ with $C_{1}(s)$ and $C_{2}(s)$ respectively, and the experimental static errors are neglected and belong to the required interval $|\varepsilon| \leq 1 \%$. The oscillatory motions of the experimental behaviors (see Fig. 5) are due to the absence of the derivative action in the implemented controller (22). These oscillatory motions have no effect in our application.

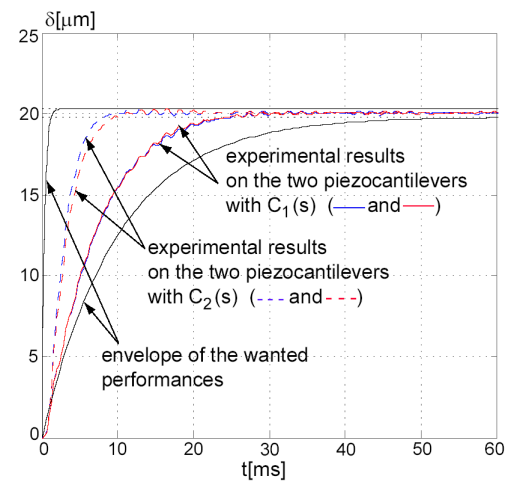

Fig. 5. Step response of the (envelope of the) reference interval model and experimental results on the two piezocantilevers using $C_{1}(s)$ and $C_{2}(s)$.

Next, a harmonic analysis is performed. For that, a sine reference input of $10 \mu \mathrm{m}$ amplitude and with different frequencies is applied. The two controllers $C_{1}(s)$ and $C_{2}(s)$ are 
still used to control both piezocantilevers. The magnitudes from the experimental results of the closed-loop piezocantilevers are plotted in (Fig. 6). The envelope magnitude of the reference $[H](s,[K],[\tau])$ is also plotted in the same graph. The different plots show that the initial resonance frequency was clearly damped and the new (experimental) magnitudes become bounded by the reference envelope, except in high frequencies because of the neglected high frequency dynamics in the model. If the specifications require the consideration of these high frequencies, the model $[G]$ of the actuators should have a higher order.

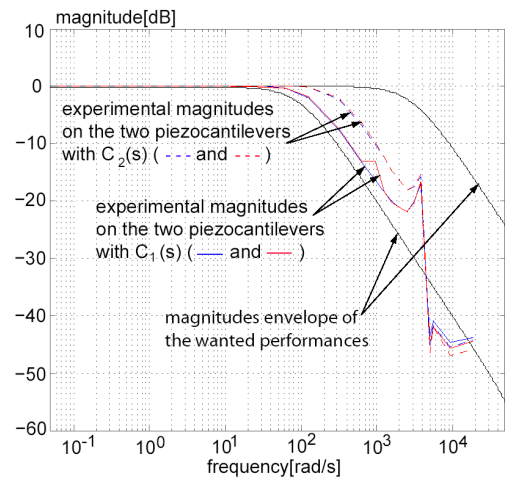

Fig. 6. Magnitudes of the wanted interval model compared with the experimental results using $C_{1}(s)$ and $C_{2}(s)$.

The last experiment consists in performing a tracking test for the two piezocantilevers using one of the controllers $C_{1}(s)$ and $C_{2}(s)$. Fig. 7 shows the experimental results when an aleatory and more smooth reference trajectory is applied. As shown in the figure, the two piezocantilevers controlled by $C_{1}(s)$ well track the reference input.
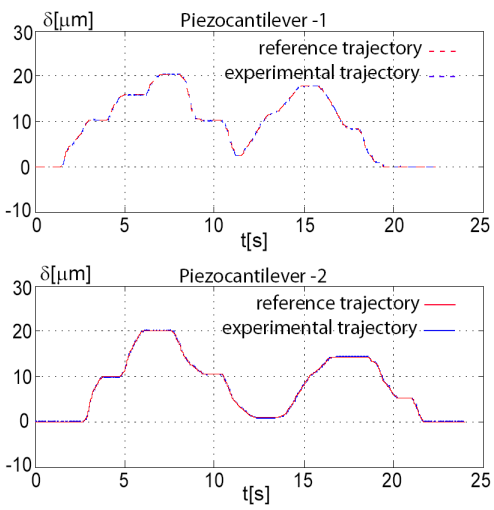

Fig. 7. Tracking performance for the two piezocantilevers when using $C_{1}(s)$.

\section{PERFORMANCE ANALYSIS OF THE CLOSED-LOOP}

In this part, we demonstrate by means of $H_{\infty}$ approach that the synthesized controllers (28) ensure the performance for the interval model defined in (21).

The $H_{\infty}$ synthesis consists to compute a robust controller that ensures given specifications by using the small-gain theorem. These specifications are transcribed into weighting functions during the synthesis. The standard $H_{\infty}$ problem consists therefore to compute a controller $C(s)$ such that [20]:

$$
\left\|F_{l}(P(s), C(s))\right\|_{\infty} \leq \gamma
$$

where $F_{l}(P(s), C(s))$ is the transfer of the interconnection between $C(s)$ and an augmented plant $P(s)$. The augmented plant $P(s)$ contains the system to be controlled $G(s)$ and the weightings that transcribe the required specifications. If there exists a controller $C(s)$ for which (29) holds, such that $\gamma \leq 1$, the specifications will be ensured.

In our case, the controller is known: $C(s)=C_{1}(s)$ first, and then $C(s)=C_{2}(s)$. The system to be controlled is interval: $[G](s)$. Finally, the specifications are given in Section IV-D and thus weighting functions can be derived. Since the system is interval, the augmented plant will also be interval: $[P](s)$. Let us consider $\gamma=1$, so if

$$
\left\|F_{l}([P](s), C(s))\right\|_{\infty} \leq 1
$$

then the controller $C(s)\left(C_{1}(s)\right.$ and $\left.C_{2}(s)\right)$ ensures the performance for any $G(s)$ inside $[G](s)$.

Fig. 8-a presents the closed-loop scheme where one weighting function $W_{1}(s)$ is sufficient to define the specifications given in Section IV-D. Fig. 8-b presents the corresponding standard scheme.

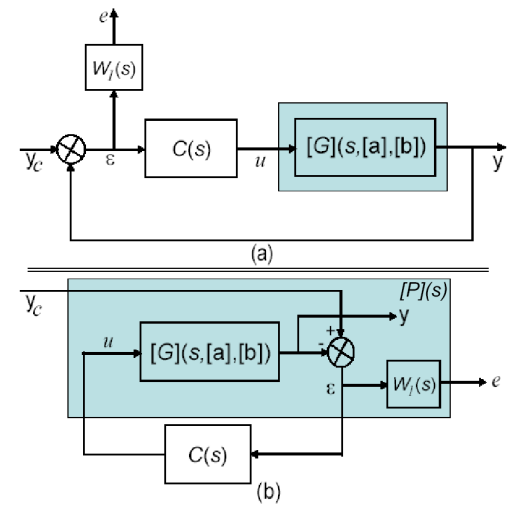

Fig. 8. Closed-loop scheme.

From Fig. 8, we have:

$$
F_{l}([P](s), C(s))=W_{1}(s)[S](s)
$$

where $[S](s)=(1+C(s)[G](s,[a],[b]))^{-1}$ represents the interval sensitivity function.

Applying the $H_{\infty}$ standard problem in (30) to (31), we obtain the following condition to be satisfied:

$$
\left\|W_{1}(s)[S](s)\right\|_{\infty}<1
$$

If we have:

$$
|[S](s)|<\left|\frac{1}{W_{1}(s)}\right|
$$

the condition (32) is verified.

From condition (33), we derive:

$$
\|[S](s)\|_{\infty}<\left\|\frac{1}{W_{1}(s)}\right\|_{\infty}
$$

Let us now give the weighting $W_{1}(s)$. For that, based on the specifications we propose: 


$$
W_{1}(s)=\frac{\frac{1}{w_{p}} s+1}{\frac{|\varepsilon|^{+}}{w_{z}} s+|\varepsilon|^{+}}
$$

where

$$
w_{z}=\frac{3}{t r^{+} \sqrt{\left(\frac{1}{|\varepsilon|^{+}}\right)^{2}-1}} \text { and } w_{p}=w_{z} \sqrt{\left(\frac{1+D \%}{|\varepsilon|^{+}}\right)^{2}-1}
$$

where $D \%$ represents the allowed overshoot.

Using the numerical values, $W_{1}^{-1}(s)$ can be written as follows:

$$
\frac{1}{W_{1}(s)}=\frac{s+1}{s+100}
$$

whose the norm is $\left\|\frac{1}{W_{1}(s)}\right\|_{\infty}=1$.

Next step consists to compute the $H_{\infty}$ norm of the sensitivity function $[S](s)=(1+C(s)[G](s,[a],[b]))^{-1}$ which is an interval transfer function. This step is performed for both $C(s)=C_{1}(s)$ and $C(s)=C_{2}(s)$. For an interval system the maximal $H_{\infty}$ norm of its sensitivity function is achieved at twelve Kharitonov vertices [21]. Based on this result, we obtain: $\left\|\left[S_{1}\right](s)\right\|_{\infty}=0.9856$ and $\left\|\left[S_{2}\right](s)\right\|_{\infty}=0.9693$, for $C(s)=C_{1}(s)$ and $C(s)=C_{2}(s)$ respectively.

From these results, we obtain $\left\|\left[S_{1}\right](s)\right\|_{\infty}<\left\|\frac{1}{W_{1}(s)}\right\|_{\infty}$ and $\left\|\left[S_{2}\right](s)\right\|_{\infty}<\left\|\frac{1}{W_{1}(s)}\right\|_{\infty}$ which mean that the specifications were effectively ensured by the controllers $C_{1}(s)$ and $C_{2}(s)$.

In order to show graphically the satisfaction of the condition (33), we plot the envelope magnitudes of $[S](s)=(1+$ $C(s)[G](s,[a],[b]))^{-1}$ (for $C(s)=C_{1}(s)$ and $C(s)=C_{2}(s)$ ) and the singular value of $\frac{1}{W_{1}(s)}$. Fig. 9 presents the results and confirms the expected results.

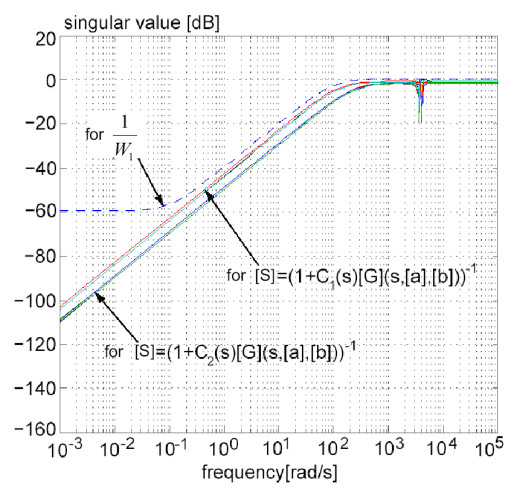

Fig. 9. Magnitudes of the envelope of $\left[S_{1}\right]=\left(1+C_{1}(s)[G](s,[a],[b])\right)^{-1}$, $\left[S_{2}\right]=\left(1+C_{2}(s)[G](s,[a],[b])\right)^{-1}$ and singular value of $\frac{\gamma}{W_{1}(s)}$.

\section{CONCLUSION}

The modeling and control of piezoelectric microactuators (piezocantilevers) were addressed in this paper. These microsystems are characterized by uncertain parameters models and need convenient modeling and robust control laws to ensure the required performance in micromanipulation, microassembly and micropositioning tasks.

In order to consider the uncertain parameters on the models of the microactuators, interval numbers are used. The main advantage is the ease and natural way to bound these uncertainties. Afterwards, we proposed a new approach to design robust controllers that can ensure the performance for the interval model. The approach provides low-order controllers that are convenient for real-time embedded systems. While the experimental results confirmed the efficiency of the proposed approach, we also used the $H_{\infty}$ technique to prove numerically the robustness of the designed controllers. The proposed design method can also be applied to other SISO systems that are characterized by parametric uncertainties.

\section{REFERENCES}

[1] Y. Haddab, N. Chaillet and A. Bourjault, 'A microgripper using smart piezoelectric actuators', IEEE/RSJ International Conference on Intelligent Robot and Systems (IROS), Takamatsu - Japan, 2000.

[2] J. Agnus, J. M. Breguet, N. Chaillet, O. Cois, P. de Lit, A. Ferreira, P. Melchior, C. Pellet and J. Sabatier, 'A smart microrobot on chip: design, identification and modeling $\breve{S}$, IEEE/ASME AIM, Kobe Japan, pp.685690, 2003.

[3] S. Devasia, E. E. Eleftheriou, R. Moheimani, 'A survey of control issues in nanopositioning', IEEE Transactions on Control System Technology (T-CST), Vol.15, No15, pp.802-823, 2007.

[4] A. Sebastian, A. Pantazi, S. O. R. Moheimani, H. Pozidis, E. Eleftheriou, 'Achieving Subnanometer Precision in a MEMS-Based Storage Device During Self-Servo Write Process', IEEE Transactions on Nanotechnology, Volume 7, Number 5, 586-595, 2008

[5] M. Rakotondrabe, Y. Haddab and P. Lutz, 'Modelling and Robust Position/Force Control of a Piezoelectric Microgripper', IEEE - International Conference on Automation Science and Engineering (CASE), 39-44, Scottsdale AZ USA, 2007.

[6] R. E. Moore, 'Interval Analysis', Prentice-Hall, Englewood Cliffs N. J., 1966.

[7] L. Jaulin and E. Walter, 'Set inversion via interval analysis for nonlinear bounded-error estimation', Automatica, 29(4), 1053-1064, 1993.

[8] L. Jaulin, 'Interval constraint propagation with application to boundederror estimation', Automatica, 36, 1547-1552, 2000.

[9] L. Jaulin, M. Kieffer, O. Didrit, and E. Walter, 'Applied Interval Analysis'. Springer, 2001.

[10] E. Walter, L. Jaulin, 'Guaranteed characterization of stability domains via set inversion', IEEE Trans. on Autom. Control, 39(4), 886-889, 1994.

[11] V.L. Kharitonov, 'Asymptotic stability of an equilibrium position of a family of systems of linear differential equations'. Differential'nye Uravnenya, 14, 2086-2088.

[12] Ye. Smaginaa, Irina Brewerb, 'Using interval arithmetic for robust state feedback design', Systems and Control Letters 46, 187-194, 2002.

[13] J. Bondia, M. Kieffer, E. Walter, J. Monreal and J. Pict'o, 'Guaranteed tuning of PID controllers for parametric uncertain systems', IEEE Conference on Decision and Control, 2948-2953, 2004

[14] C.-T. Chen, M.-D. Wang, 'A two-degrees-of-freedom design methodology for interval process systems', Computers and Chimical Engineering, 23,1745-1751, 2000.

[15] K. Li, Y. Zhang, 'Interval Model Control of Consumable DoubleElectrode Gas Metal Arc Welding Process', IEEE - Transactions on Automation Science and Engineering (T-ASE), 10.1109/TASE, 2009.

[16] Keel, L. H., Bhattacharyya, S. P., "Control system design for parametric uncertainty", International Journal of Robust and Nonlinear Control, Vol.4, 87-100, 1994.

[17] S. Khadraoui, M. Rakotondrabe and P. Lutz, "Robust control for a class of interval model: application to the force control of piezoelectric cantilevers", IEEE - CDC, (Conference on Decision and Control), accepted, Atlanta Georgia USA, December 2010.

[18] Micky Rakotondrabe, Yassine Haddab and Philippe Lutz, 'Quadrilateral modelling and robust control of a nonlinear piezoelectric cantilever', IEEE - Transactions on Control Systems Technology (T-CST), Vol.17, Issue 3, pp:528-539, May 2009.

[19] Ljung, L. "System identification: Theory for the user", 2nd ed, PTR Prentice Hall, Upper Saddle River, N.J., 1999.

[20] Balas, G. J., Doyle, J. C., Glover, K., Packard, A. and Smith, R., “ $\mu$ -synthesis and synthesis toolbox", The Mathworks User's Guide-3, 2001

[21] Wang, L. " $H_{\infty}$ Performance of Interval Systems", eprint arXiv:math/0211013, Vol.1, 1-8, 2002. 